\title{
Positioning and Tracking Performance Analysis of Hypersonic Vehicle Based on Cubature Kalman Filter
}

\author{
Jianghui Zeng ${ }^{1,}$, , Yongming $\mathrm{Gao}^{2}$ \\ ${ }^{1}$ Graduate School of Space Engineering University, Beijing 101416, China; \\ ${ }^{2}$ Aerospace Information Institute of Space Engineering University, Beijing 101416, China. \\ a18822686122@163.com
}

\begin{abstract}
Aiming at the problem of location and tracking of hypersonic vehicles in adjacent space, the tracking and tracking performance of low-track binary satellites based on targets is explored. The trajectory model of hypersonic vehicles is established. Based on STK and Matlab simulation software, two stars are built for the target. The tracking and observation scenes are located. The classical kinematics model is used to track the target. Cubature Kalman tracking algorithm based on CV, CA and CS models is studied. The comparison analysis of three tracking algorithms is carried out. The simulation results show that the CS and CA models are more suitable for the positioning and tracking of hypersonic vehicles than the CV model. At the same time, it is pointed out that to further improve the target tracking performance, a maneuver detection or multi-model algorithm is needed.
\end{abstract}

Keywords: LEO, Hypersonic vehicle, Double star positioning tracking, Kinematic model, CKF.

\section{Introduction}

The adjacent space generally refers to the height of the ground from 20 to $100 \mathrm{~km}$, which is between the airspace of traditional aircraft such as the airspace of civil aviation aircraft and the orbit of the near-Earth satellite [1]. It has always been "no one cares", with the major powers. with the continuous development of aerospace technology and the continuous improvement of the cognitive level of the adjacent space environment, all major military powers are now turning their attention to this airspace. The spacecraft can be divided into high-speed aircraft and low-speed aircraft according to the speed of operation. Among them, the target that can fly at a speed not lower than Mach 5 in the atmosphere or across the atmosphere is called a hypersonic vehicle [2]. Fast flight speed, high cruising altitude, strong maneuverability, strong penetration capability, fast and accurate strike, long-range fast delivery, etc., will become the weapon for completing anti-interventional operations, accurately combating time-sensitive targets and major strategic nodes in the future. This poses a major strategic threat to China's national defense security.

The world's old military powers, the United States has a strong military industry and national defense research capabilities, the development of many advanced, high performance supersonic aircraft, such as the use of boost-gliding ballistic flight HTV-2 hypersonic aircraft [3] The X-51A hypersonic vehicle with boost-cruise flight mode is currently actively developing other more advanced hypersonic vehicles.

The location tracking of the target is the key to air defense and anti-missile warfare, but the current early warning detection equipment-ground warning radar is greatly affected by various factors for the early warning detection capability of such targets, due to the current flight of hypersonic vehicles. The height is lower than that of the traditional ballistic missile. The ground tracking station's positioning and tracking of the target is greatly affected by the curvature of the earth [4]. Due to the weather conditions and geographical location, the warning time for hypersonic vehicles is extremely limited. Since the hypersonic vehicle itself is a small target during the movement, its RCS characteristics change drastically [5]. During hypersonic flight, a plasma sheath is generated around the fuselage, which forms a scattering effect on the radar wave, making the ground radar for hypersonic speed. Positioning and tracking of aircraft is more difficult. Therefore, the space-based low-orbit binary star system is a better choice for solving the problem of positioning and tracking of near-space hypersonic vehicles. 
At present, there are many literatures on the location and tracking of hypersonic boost-glide hypersonic vehicles. Many experts and scholars have given their own answers. Among them, Zhang Yuan, $\mathrm{Wu}$ Hao [6] and others are studying adjacent space hypersonic vehicles. After the motion process, the CV-CA-Singer interactive multi-model algorithm is proposed, but the target tracking performance is insufficient. It is only suitable for hypersonic targets when the maneuver is insufficient. The hypersonic vehicle is known for its maneuverability. For example, the HTV-2 hypersonic vehicle can move in the atmosphere for about 3000s [7] in its jumping gliding section, so it is necessary to have the early warning detection and location tracking capability of the target jump-gliding segment. Zhang Xiangyu [8] et al. proposed an interactive multi-model algorithm based on sinusoidal models with different angular rates for the target orientation tracking from the improved direction of the target motion state model. The algorithm improves the hypersonic speed for adjacent spaces. The locating and tracking performance of the gliding-flying ballistic target, but there are many prior parameters in the model, and the uncertainty of the parameter design accuracy will inevitably affect the positioning and tracking performance of the target. In the traditional positioning tracking of ballistic missile targets, the filtering algorithm often uses Extended Kalman filter (EKF), but there is a first-order truncation error in the algorithm, which makes the algorithm's positioning tracking accuracy for the hypersonic vehicle with frequent maneuvering. Significant decline. Qin Lei [9] et al. designed a tracking Kalman filter based on the Singer model and obtained good results.

\section{Target Tracking Observation Scene Design}

\subsection{Target Aircraft Ballistic Model Construction}

To achieve the positioning and tracking of the hypersonic vehicle, the observation scene of the target must be designed. STK software is a kind of software commonly used in aerospace simulation. The article will use STK and Matlab simulation software for simulation analysis.

First, to perform the target tracking, it is necessary to first analyze the kinematics of the hypersonic boost-glider to find a kinematic model that matches the motion state of the target. Near-space hypersonic vehicles can be maneuvered by controlling the angle of attack and the angle of inclination. At present, hypersonic vehicles can be maneuvered in several ways [10]:

1. Maintain the same flight altitude and maximize lateral maneuver

2. Wait for dynamic pressure flight, the inclination angle is constant

3. The angle of attack maintains the maximum lift-to-drag ratio, and the tilt angle is constant.

4. The angle of attack is constant and the angle of inclination is also constant.

In this paper, the aircraft angle of attack design in [11] is used to obtain the target ballistic data based on the fourth-order Runge-Kutta iteration. Based on the Earth's inertial coordinate system, the equation of motion of the target aircraft can be expressed as:

$$
\begin{aligned}
\frac{d r}{d t} & =V \sin \gamma, \\
\frac{d \theta}{d t} & =\frac{V \cos \gamma \cos \psi}{r \cos \phi}, \\
\frac{d \phi}{d t} & =\frac{V \cos \gamma \sin \psi}{r}, \\
\frac{d V}{d t} & =-\frac{D}{m}-\frac{\mu}{r^{2}} \sin \gamma, \\
\frac{d \gamma}{d t} & =\frac{1}{V}\left(\frac{L}{m} \cos \sigma+\left(\frac{V^{2}}{r}-\frac{\mu}{r^{2}}\right) \cos \gamma\right), \\
\frac{d \psi}{d t} & =\frac{1}{V}\left(\frac{L}{m} \frac{\sin \sigma}{\cos \gamma}-\frac{V^{2}}{r} \cos \gamma \cos \psi \tan \phi\right)
\end{aligned}
$$


Among them, $r$ is the distance between the center of mass of the aircraft and the center of mass of the earth; $\theta$ is the longitude of the target; $\phi$ is the latitude of the target; $V$ is the flight rate of the aircraft; $\gamma$ is the local ballistic inclination; $\psi$ is the east-north angle of the velocity vector. What appears in the equation is a constant called the Earth's gravitational constant, and its value is $\mu=3.986005 \times 10^{14} \mathrm{~m}^{3} / \mathrm{s}^{2}$.

Among them, $D$ and $L$ are the resistance and lift experienced during the movement of the hypersonic vehicle. The expressions are as follows:

$$
\begin{aligned}
& D=C_{D}(\alpha, \text { Ma }) \frac{\rho}{2} V^{2} S \\
& L=C_{L}(\alpha, \text { Ma }) \frac{\rho}{2} V^{2} S
\end{aligned}
$$

Equation (2) is the local atmospheric density in the motion of the aircraft. It can be fitted to the atmospheric density of the target and the local sound velocity according to the 1976 US standard atmospheric model. The obtained atmospheric density function is:

$$
\rho=\rho_{0} e^{-k h}
$$

Where $\rho_{0}$ is the sea level standard atmospheric pressure, $k=-0.1133$ is a constant, $h$ is the height of the target, whose unit is kilometers, and respectively the resistance coefficient $C_{D}$ and the lift coefficient $C_{L}$ of the aircraft. These two parameters are functions of the angle of attack and the target flying Mach number. The functions of the lift coefficient and the drag coefficient are expressed as [12]:

$$
\begin{aligned}
& C_{D}=C_{D 0}+C_{D 1} \alpha^{2}+C_{D 2} \exp \left(C_{D 3} M a\right) \\
& C_{\mathrm{L}}=C_{\mathrm{L} 0}+C_{\mathrm{L} 1} \alpha+C_{L 2} \exp \left(C_{\mathrm{L} 3} M a\right)
\end{aligned}
$$

Among the formula:

$$
\begin{aligned}
& C_{D 0}=0.024, C_{D 1}=7.24 e-4, \\
& C_{D 2}=0.406, C_{D 3}=-0.323, \\
& C_{\mathrm{L} 0}=-0.2317, C_{\mathrm{L} 1}=0.0513, \\
& C_{L 2}=0.2945, C_{\mathrm{L} 3}=-0.1028
\end{aligned}
$$

\subsection{Double Star Passive Angle Measurement}

Since both infrared warning satellites are equipped with an infrared tracking sensor, the target can be tracked for a period. At the same time, because the distance between the target aircraft and the satellite is far, sometimes even thousands of kilometers can be reached. Currently, the target is only one-pixel point on the infrared sensor of the satellite [13]. To facilitate the description of the target observation angle, it is assumed that the orbit coordinate system of the satellite coincides with the observation coordinate system of the sensor mounted on the star, and the definition of the observation coordinate system is as follows:

The center of mass of the observation star is the origin of the coordinate system. The axis $Z_{0}$ is pointed by the centroid of the satellite to the center of mass of the earth (also known as the local vertical line). The axis $Y_{0}$ is the negative normal direction of the orbit of the satellite. The axis $X_{0}$ 
and the other two axes form the right-handed coordinate system. Figure 1 shows a schematic diagram of satellite angle measurement.

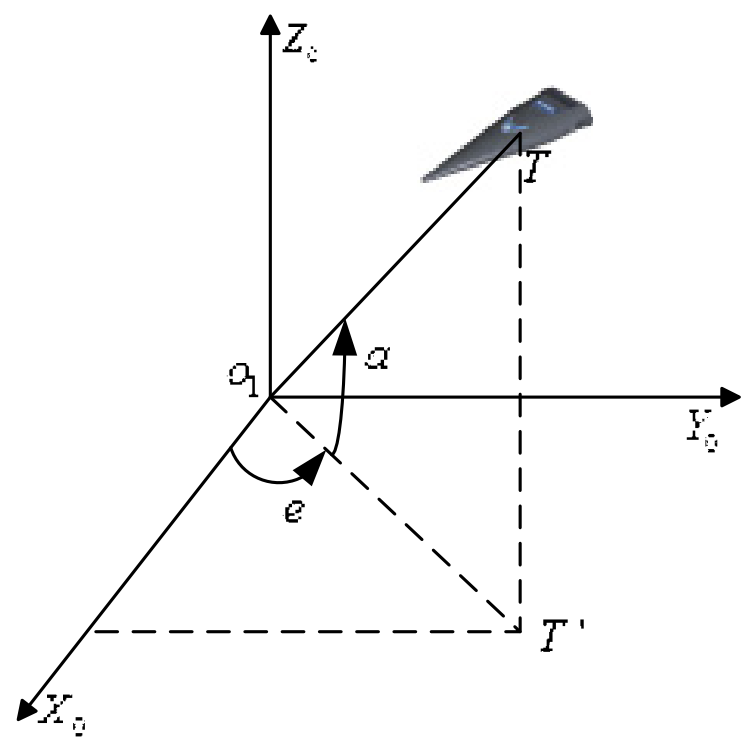

Fig. 1 Satellite angle measurement diagram

(1) Azimuth angle $e$, the angle between the projection of the satellite's line of sight vector in the plane $X_{0} Y_{0} O_{1}$ and the coordinate axis $X_{0} O_{1}$, which is positive by pointing from $X_{0} O_{1}$ to $O_{1} T^{\prime}$, and its value range is $[0, \pi]$.

(2) Pitch angle $a$, the angle between the projection $O_{1} T^{\prime}$ of the satellite line-of-sight vector in the plane $X_{0} Y_{0} O_{1}$ and the line of sight $O_{1} T$, which is positive by pointing from $O_{1} T$ 'to $O_{1} T$, and its value range is $\left[-\frac{\pi}{2}, \frac{\pi}{2}\right]$.

\section{Hypersonic Vehicle Positioning Tracking}

At present, there are many literatures on conventional maneuvering target tracking technology, and the target location tracking problem has always been the focus of research. The location tracking problem of hypersonic vehicles using the jump-gliding flight mode is a completely new problem in maneuvering target tracking.

The target tracking of the target mainly includes such aspects as the design of the tracking sensor suitable for different characteristic targets, the classical motion state model of the target, the nonlinear filtering, the target maneuver detection, the multi-model algorithm, etc., which appear in these fields. Many theoretical results.

At present, there are two basic ideas for the trajectory tracking of the target. One is based on the dynamic principle of the target, the dynamic modeling of the target or the fitting of the characteristic parameters, and the target ballistic inverse design is carried out by using the constraints in the flight process. This kind of method is very difficult to implement under the condition that the defender has no prior information of the target. For the passive defender, the observation of the target can only obtain information such as angle, distance, Doppler frequency, etc. The target model, performance parameters and other content are difficult to get a clear judgment. Here, the coordination of other observation information is needed. Another way is to realize the target tracking based on the traditional kinematics model. This method does not require many target tracking. The a priori information needs to obtain continuous tracking observation information for the target, but this method has the problem that the accuracy of the target positioning is insufficient due to inaccurate model fitting. In this paper, the second idea is chosen. Firstly, the mature target kinematics model is applied to explore the motion characteristics of various kinematic models in fitting hypersonic 
vehicles. In the traditional target tracking algorithm, there are several kinematic models that are often used, such as uniform motion (CV) model, uniform acceleration motion (CA) model, Singer model, current statistical model (CS) [13], Jerk model, sinusoidal model, etc. In these motion models, three models of CV, CA and CS are selected for comparative simulation analysis. The results obtained after simulation analysis can provide reference for model set design for the interactive multi-model (IMM) algorithm to be used later. The system dimensions corresponding to these kinematic models are 6dimensional, 9-dimensional, and 9-dimensional, respectively.

Set the current state value of the target to $X_{k}$, which contains the position, velocity, acceleration and other information of the target in the $\mathrm{X}$-axis, $\mathrm{Y}$-axis, and $\mathrm{Z}$-axis directions, the discrete state transition matrices of the above three models are different, and different state transition matrices are set, wherein the state transition equation of the model Can write:

$$
X_{k}=\phi_{i} X_{k-1}+W_{k}
$$

In the above formula (6), $X_{k-1}$ is the motion state of the aircraft at the last moment, $W_{k}$ is the state noise, and the covariance of the state noise is $Q_{k}, Q_{k}=E\left(W_{k} W_{k}^{T}\right)$, where in each model corresponds to a different one.

Set the position of the target on the observation coordinate system on the first satellite, and according to the azimuth and elevation angles described above, the observation equation of the binary star for the target can be obtained:

$$
Z=\left[\begin{array}{l}
e_{1} \\
a_{1} \\
e_{2} \\
a_{2}
\end{array}\right]=\left[\begin{array}{c}
\arctan \frac{y_{t 1}}{x_{t 1}} \\
\arctan \frac{z_{t 1}}{\sqrt{x_{t 1}^{2}+y_{t 1}^{2}}} \\
\arctan \frac{y_{t 2}}{x_{t 2}} \\
\arctan \frac{z_{t 2}}{\sqrt{x_{t 2}^{2}+y_{t 2}^{2}}}
\end{array}\right]+v
$$

In the above formula (7), $\mathrm{Z}$ is the observation of the target by the binary star; $v$ is the measurement noise; the Gaussian white noise is assumed to be zero mean, and the target $X=[x, y, z]^{T}$ is in the position of the Earth's inertial coordinate system (ECI), and the binary star is observed in the Earth's inertial coordinate system ( ECI) location is $X_{s i}=\left[x_{s i}, y_{s i}, z_{s i}\right]^{T}(i=1,2)$. The relationship is as follows:

$$
\left[\begin{array}{c}
x_{t i} \\
y_{t i} \\
z_{t i}
\end{array}\right]=T_{E C I i}^{o r b}\left[\begin{array}{c}
x-x_{s i} \\
y-y_{s i} \\
z-z_{s i}
\end{array}\right]
$$

Among them, $T_{E C I i}^{o r b}(i=1,2)$ is the coordinate transformation matrix of the Earth's inertial coordinate system to the satellite's observation coordinate system for the target, which is the coordinate transformation matrix of satellite velocity and position, which is described in detail in [14]. The above two formulas constitute the observation system of the binary star for the target.

In the whole nonlinear system, nonlinear filtering is the key to the whole positioning and tracking system. Because of the strong nonlinearity of the observation of hypersonic vehicles, the nonlinear 
Kalman filter must be selected, in which the Cubature Kalman filter Compared with other filters, the filter has no higher-order truncation error in the filtering operation of the Extended Kalman filter, and the performance of the Unscented Kalman filter in the face of higher-order nonlinear systems. Unsatisfactory, the Cubature Kalman filter is more stable and performs better than the unscented Kalman filter. The Cubature Kalman filter uses a third-order spherical radial volume criterion to approximate the specific process of the Gaussian filtering algorithm, including the initialization, time update, and status update of Cubature Kalman filter.

\section{Simulation Analysis}

\subsection{Target Aircraft Ballistic Simulation}

In this paper, to locate and track the typical trajectory of the jump-gliding hypersonic vehicle, the initial flight state value of the target aircraft is set, and the flight state is simulated and analyzed. Using the commonly used fourth-order Runge-Kutta method to perform the iteration of the motion state [15], the motion state of the target aircraft in 2000s is obtained. Although the fourth-order Runge-Kutta method is slightly complicated, the iterative precision of the method is better. High, set the initial flight height of the target aircraft to $50 \mathrm{~km}$, the initial latitude and longitude is 0 degrees, the target initial speed is $6000 \mathrm{~m} / \mathrm{s}$, and the initial ballistic inclination and ballistic yaw angle of the target are $-0.1 \mathrm{rad}$ and $-\frac{\pi}{6} \mathrm{rad}$, for the formula (1) The simulated trajectory after the fourth-order Runge-Kutta iteration is shown in Figure 2.

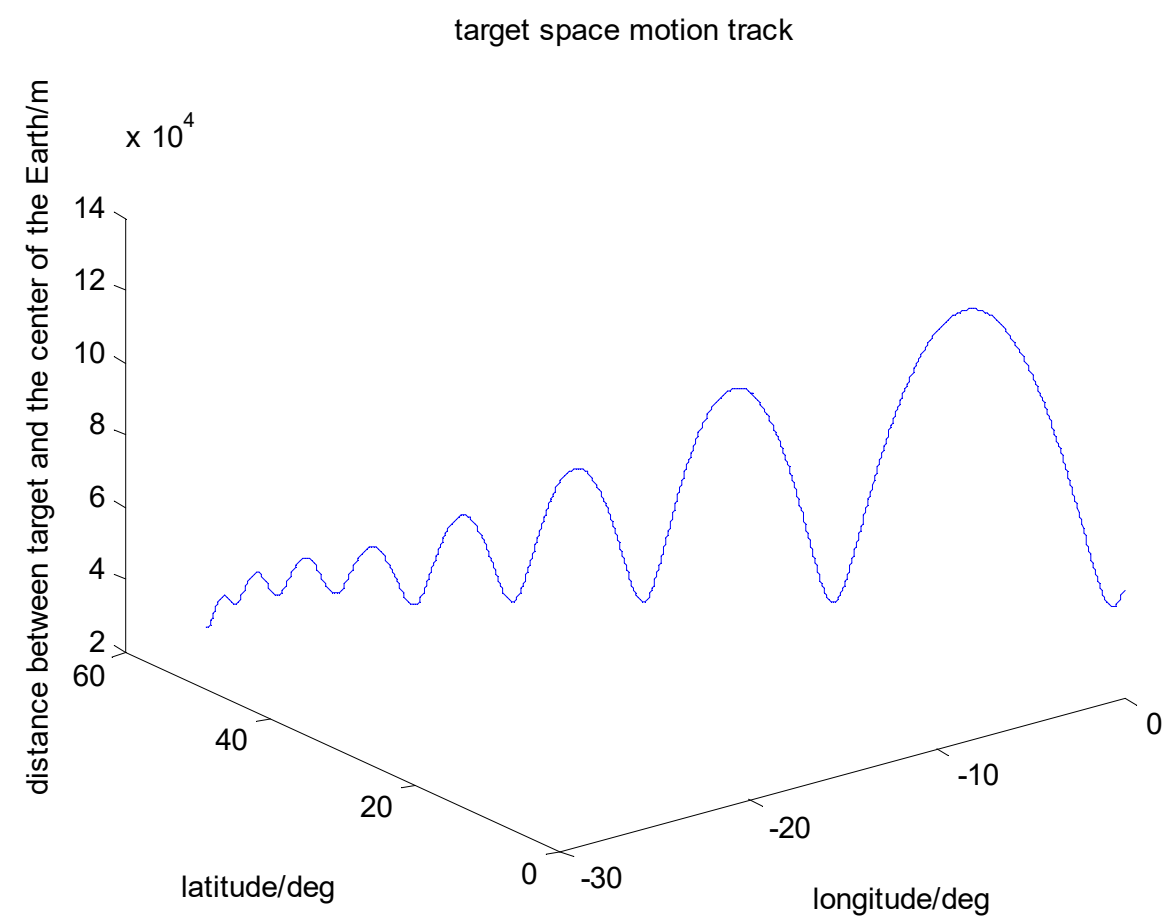

Fig 2. Target aircraft space motion track 


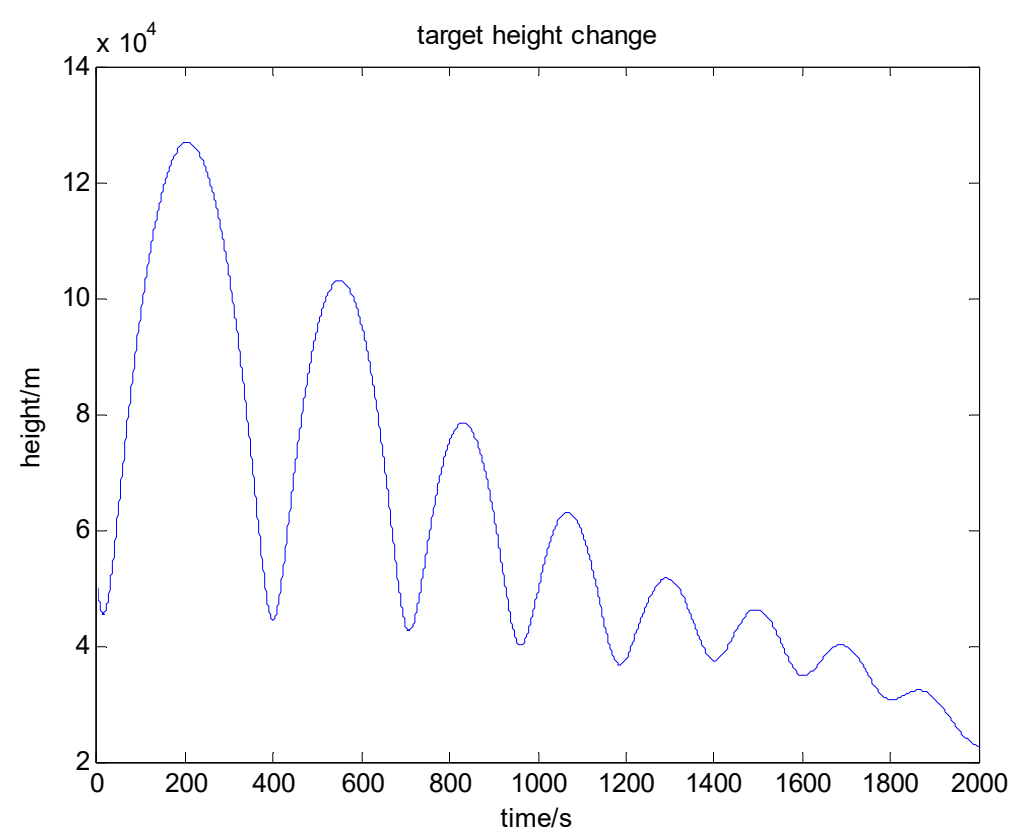

Fig 3. Target aircraft height change

The corresponding ballistic data obtained by the fourth-order Runge-Kutta method is written into the Ephemeris e file and imported into the STK, as shown in Figure 5, and based on the description of the constellation design in the US STSS satellite system in [16], in STSS. To meet the needs of global coverage, the satellite uses polar orbits with a constellation height of $1600 \mathrm{~km}$. In practice, $\mathrm{T} / \mathrm{P} / \mathrm{F}$ is used to describe the relative geometry of the constellation, where $\mathrm{T}$ is the total number of satellites in the constellation and $\mathrm{P}$ is the number of orbital orbitals. $\mathrm{F}$ is the metric of the relative phase of the satellites of adjacent orbital planes, and the value of $\mathrm{F}$ is any integer between [0, P-1]. Here, the satellite constellation configuration is set to $24 / 3 / 1$, based on this observation. The target and the corresponding data given in the STK are used for the location analysis of the target.

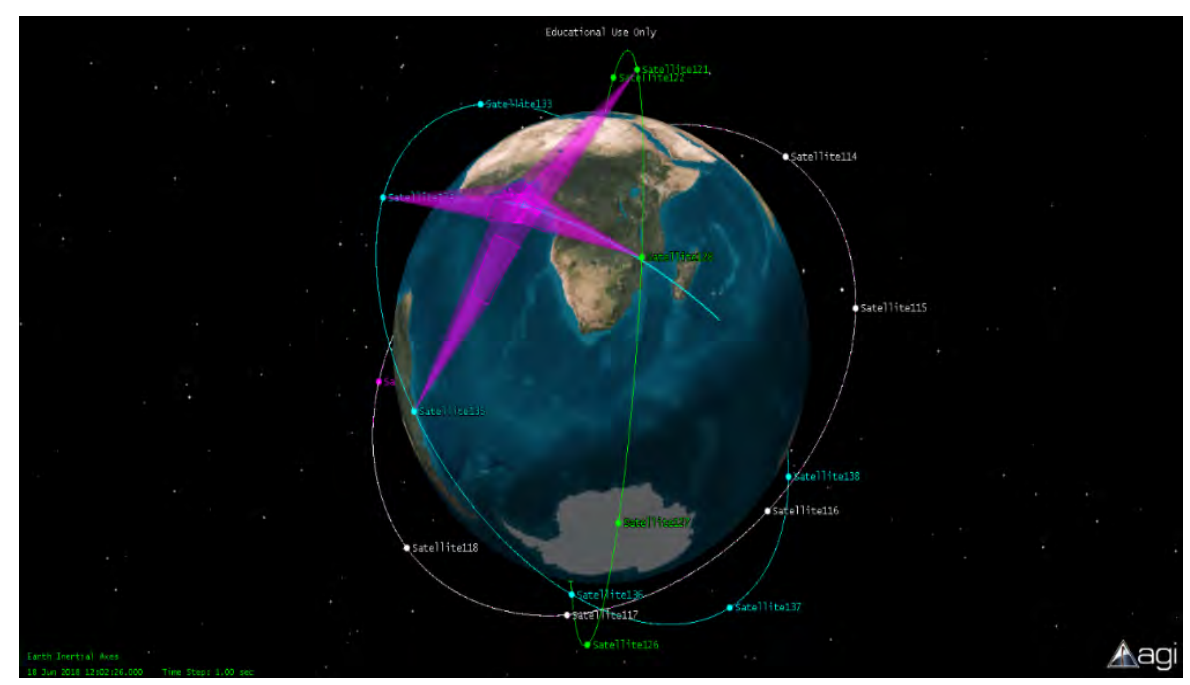

Fig 4. STK build target observation scene

\subsection{Double Star Filter Tracking Simulation}

Select two satellites, Seed32 and Seed33, in the same orbit to obtain the visible time of the two satellites. The two satellites can be seen together for 9 minutes, that is, 12:08:00-12:16:59 time for the target. The positioning and tracking performance are compared and analyzed. The total time of flight of the aircraft in space is 12:00:01-12:33:21, totaling 2001s.

Here, the tracking camera on the binary star is used for tracking observation. Since the devices on the star are optical instruments, only the above four angle measurement values can be obtained. The 
position error of the observation binary star itself is $15 \mathrm{~m}(3 \sigma)$, the speed error is $0.2 \mathrm{~m} / \mathrm{s}(3 \sigma)$, and the angle error of the device on the observation star is $\operatorname{rad}(3 \sigma)$. At the same time, to better compare the filtering performance of the three algorithms for the target state, the mean square error (RMSE) commonly used in error calculation is used. The calculation formula of RMSE is as follows:

$$
R M S E=\sqrt{\frac{1}{N} \sum_{i=1}^{N}\left[(\hat{x}-x)^{2}+(\hat{y}-y)^{2}+(\hat{z}-z)^{2}\right]}
$$

In the above formula, $\mathrm{N}$ is the number of Monte Carlo targets, $(x, y, z)$ is the real state value of the target. $(\hat{x}, \hat{y}, \hat{z})$ is the target state value obtained by filtering. After Matlab simulation experiments, the filter tracking performance of the three algorithms is obtained.

In the filtering algorithm, it is necessary to set the error of each model in each direction in advance. After theoretical analysis of the theoretical trajectory of the hypersonic vehicle, the error of each direction of the three models can be set [17], wherein the CV model is on the X axis. The noise in the $\mathrm{Y}$-axis and Z-axis directions are 35, 40, and 10, respectively. The noise of the CA model in the Xaxis, Y-axis, and Z-axis directions is $2.5,4.5$, and 1.5, respectively. The setting method of the CS model is slightly complicated. The maneuvering frequency of the CS model is set to 0.1 , and the maximum and minimum accelerations are known from the real trajectory of the target. The acceleration variance adaptive algorithm in the current model of the maneuvering target is adopted. At the same time, the initial value of the target is set to be obtained by the double star angle measurement geometric positioning algorithm. The velocity vector is obtained by the difference between the target state value and the initial value of the acceleration. The initial value of the acceleration is set to 0 , and the initial covariance matrix of the target is set to the unit matrix of the same state dimension. Each algorithm performs Monte Carlo shooting experiments 200 times, and the positioning and tracking performance of each filtering algorithm can be obtained.

Combined with the following three graphs, the overall accuracy of the CV model is not as good as the other two models. There is no obvious advantage in the RMSE of position and velocity. At the same time, the RMSE of the velocity can be seen, and the CV model has obvious fluctuations. The effect is poor. The CA model also has the problem of insufficient filtering precision. The accuracy of the CA model is like that of the CS model in the RMSE of the position. However, in the description of the target velocity, the CS model considers the influence of the current maneuvering acceleration at the speed. The accuracy is higher, and the convergence is better than the other two models. Therefore, in the positioning tracking of hypersonic vehicles, no model can describe the whole motion state of the target perfectly. These models only try to "fit" the actual trajectory of the target, but in terms of the overall performance of the motion state description. The performance of the CS and CA models is due to the CV model. At the same time, since the target is a mobile aircraft with strong maneuverability, to obtain better filter tracking performance, it is necessary to introduce maneuver detection or multi-model algorithm to further improve the target tracking accuracy.
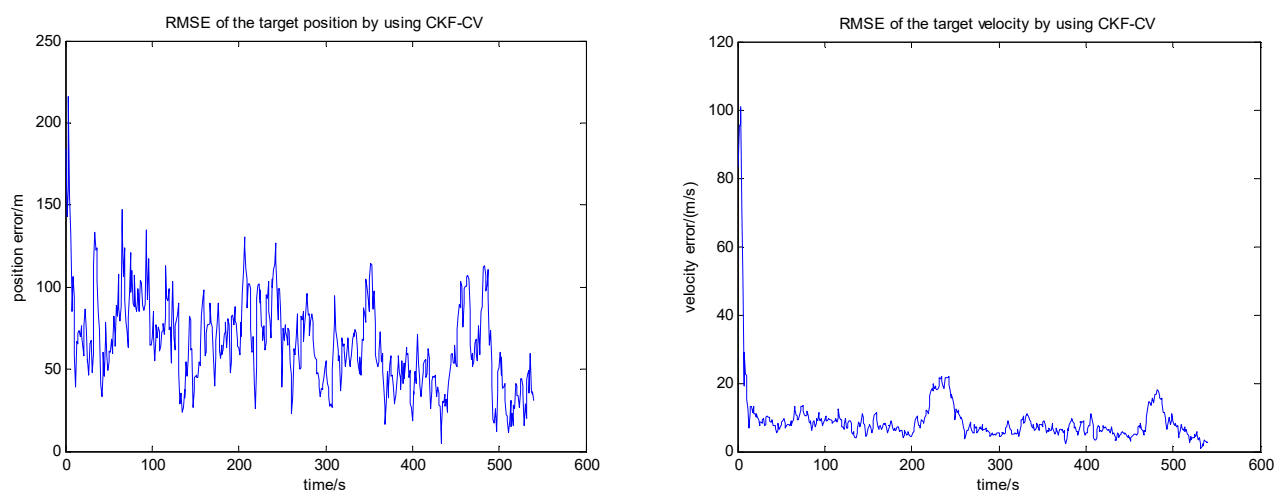

Fig 5. CKF-CV filtering to obtain position and velocity RMSE 

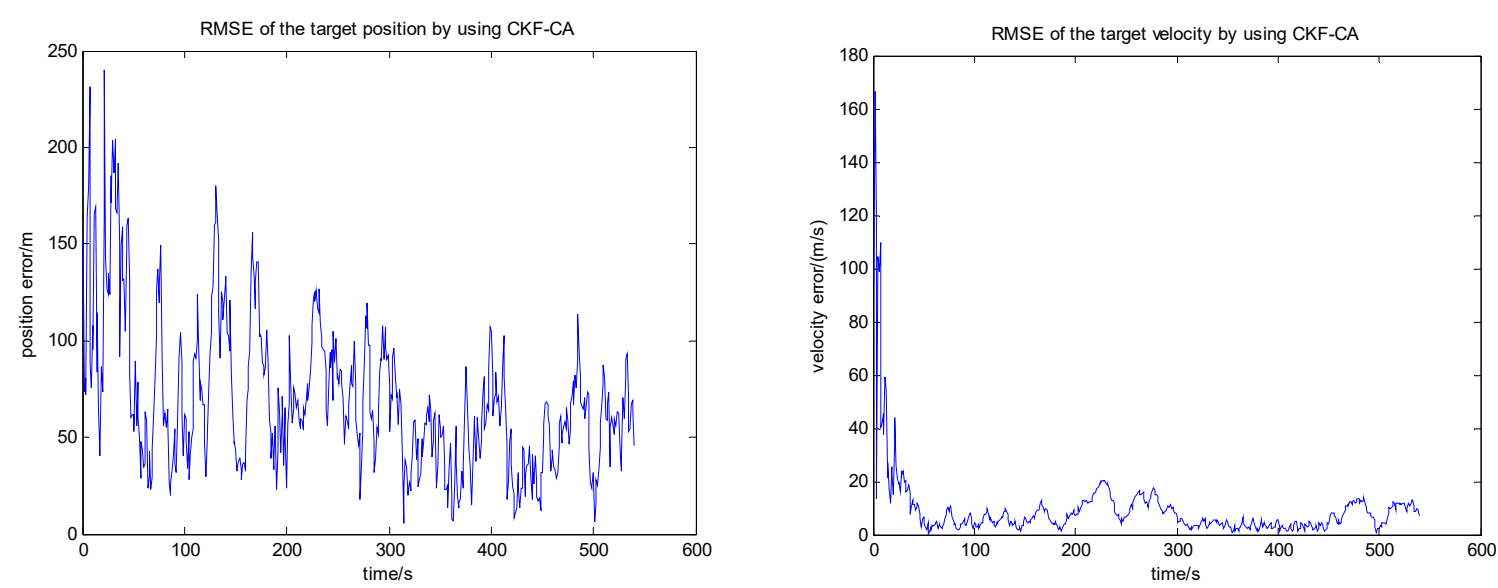

Fig 6. CKF-CA filtering to obtain position and velocity RMSE
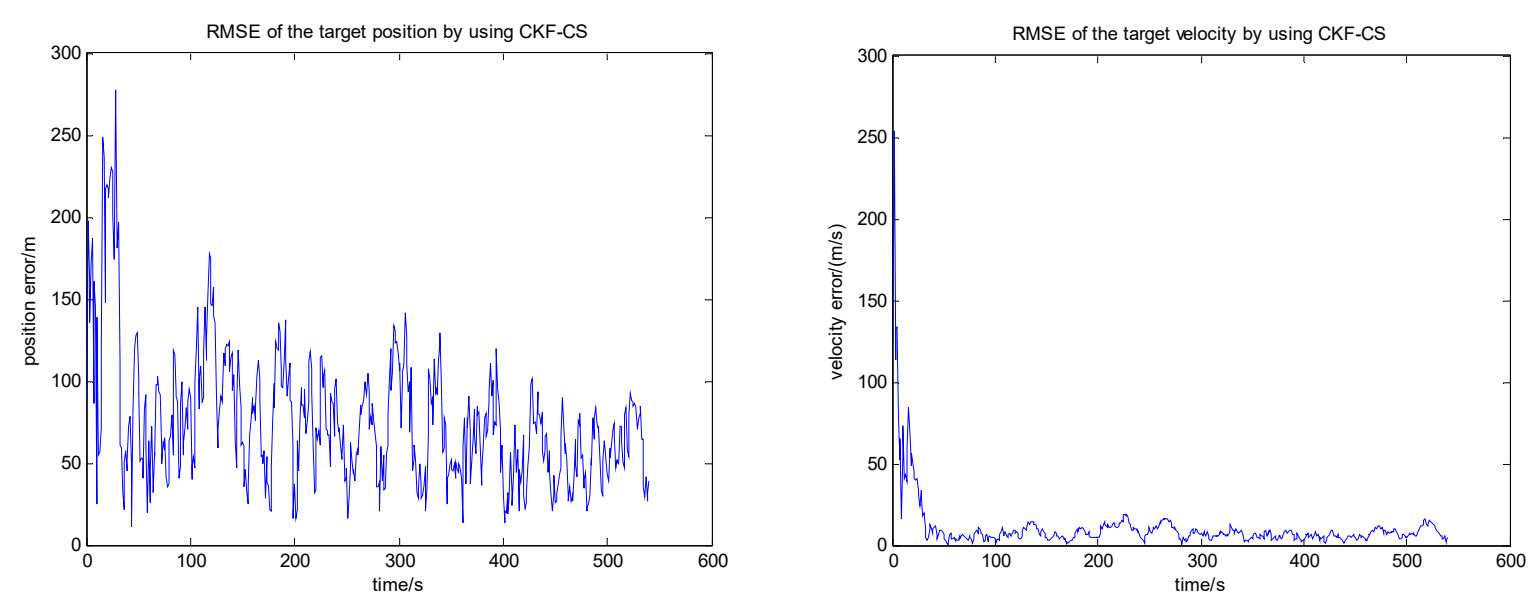

Fig 7. CKF-CS filtering to obtain position and velocity RMSE

\section{Summary}

In this paper, the problem of position and tracking of hypersonic vehicles is introduced. Firstly, the motion state model of hypersonic vehicle is introduced. Based on the fourth-order Runge-Kutta method, the target ballistic simulation is carried out, and the jump-gliding trajectory of the aircraft is obtained. At the same time, the real motion of the target aircraft is obtained. The state is written as the ephemeris e file that STK software can recognize, and the low-orbit satellite constellation is set in the STK. Based on the simultaneous visible conditions of the binary star, the comparative analysis of the positioning and tracking performance is developed. The simulation results show that the CV model has insufficient positioning and tracking ability for the target with strong maneuverability. The CA and CS models have better ability to "fit" in the motion stage of the aircraft. To further improve the target tracking accuracy, it should be introduced. The maneuver detection or multi-model algorithm further improves the positioning tracking accuracy of the target.

\section{References}

[1]. Cheng Xuan, Xiao Cunying, Hu Xiong. Research progress on the influence of adjacent atmospheric environment on the aerodynamic characteristics of hypersonic vehicles[J]. Flying Missile, 2018(5).

[2]. Huang Zhicheng. Hypersonic Weapons and Their Impact on Future Wars [J]. Tactical Missile Technology, 2018 (03): 1-7. 
[3]. Wang Lu, Xing Qinghua, Mao Yifan. Analysis of HTV-2 target characteristics [J]. Flying missile, 2014 (07): 22-24+71.

[4]. Li Luogang. Research on positioning and tracking of adjacent spacecraft and guidance of intercepting missiles [D]. Harbin Institute of Technology, 2013.

[5]. Yu Zhefeng, Liu Jiaqi, Liu Lianyuan, Ren Aimin, Liang Shichang, Chen Xuming, Qi Runhui, Huang Jie.Study on RCS Characteristics of Near Space Hypersonic Vehicles[J]. Journal of Astronautics, 2014, 35(06): 713-719.

[6]. Zhang Yuan, Wu Wei et al. Research on Modeling and Tracking Method of Moving Objects in Near Space[J]. Fire Control Radar Technology, 2013, 42(4): 22-27.

[7]. Yan Huaping, Jiang Chongwen. Overview of hypersonic technology verification aircraft HTV-2 [J]. Flying missiles, 2013 (06): 7-13.

[8]. Zhang Xiangyu, Wang Guohong, Li Junjie, Sheng Dan. Near-space hypersonic sliding trajectory target tracking technology[J]. Acta Aeronautica Sinica, 2015, 36(06): 1983-1994.

[9]. Qin Lei, Li Junlong, Zhou Wei. The problem of HTV-2 tracking filtering and prediction for nonballistic targets in adjacent space[J]. Space Control,2015,33(02):56-61+69.

[10]. CAO Yajie, LI Junlong, QIN Lei. Study on non-ballistic target tracking filtering algorithm in adjacent space[J]. Journal of System Simulation,2016,28(06):1351-1358.

[11]. Li Qiang. Research on re-entry guidance control technology for hypersonic gliding aircraft [D]. Beijing Institute of Technology, 2015.

[12]. Sun Yong, Duan Guangren, Zhang Yurui, Zhang Ze. Improved aerodynamic coefficient model for hypersonic vehicle re-entry process[J]. System Engineering and Electronics, 2011,33 (01):134-137.

[13]. Zhou Hongren et al. Maneuvering target tracking [M]. National Defence Industry Press, 1991.

[14]. Li Hengnian. The best estimation method and application of space measurement and control [M]. National Defense Industry Press, 2015.

[15]. Fan Y, Zhu W, Bai G. A Cost-Effective Tracking Algorithm for Hypersonic Glide Vehicle Maneuver Based on Modified Aerodynamic Model [J]. Applied Sciences, 2016, 6(10): 312.

[16]. Mao Yifan, Zhang Duolin, Wang Lu. Simulation Analysis of Ballistic Target Detection by STSS[J]. Infrared Technology,2015,37(03):218-223.

[17]. Liang Xiaohu, Zhu Wuxuan, Zhao Hua. Research on tracking algorithm of high-speed aircraft in adjacent space based on kinematics model[C]// China Aerospace Society Chinese Space Law Society 2012 Annual Conference. 2012. 